\title{
Comparison of normal and asthmatic circadian rhythms in peak expiratory flow rate
}

\author{
M R HETZEL AND T J H CLARK \\ From the Brompton Hospital, London
}

ABSTRACT A computer technique (cosinor analysis) has been used to evaluate circadian rhythms in airway calibre in normals and asthmatics. Two hundred and twenty-one normal subjects re-N corded peak expiratory flow rate (PEFR) at home four times a day for seven days. Rhythm detection was statistically significant in 145 of them $(65.6 \%)$ who showed a mean amplitude of $8.3 \%$ of individual mean PEFR ( \pm SD 5.2\%). Amplitude was independent of age, sex, atopy, familyo history of asthma, and smoking habit. Fifteen of them were also studied three times a day for fiveo days in the laboratory with flow-volume loops. Eleven showed significant PEFR rhythms at home. No single measurement from the flow-volume loop showed periodicity in as many of them butso rhythms were now also detected in the other four normal subjects in some components of the loop. Fifty-six asthma patients were studied with a similar protocol of PEFR measurement and compared응 with the 145 rhythmic normal subjects. Mean phases of the normal and asthmatic rhythms were not significantly different with acrophases (peak of rhythm cycle) at 1557 and 1526 respectively. Theo mean asthmatic amplitude was, however, significantly greater at $50.9 \%$. Nocturnal asthma, there-负 fore, probably represents an exaggeration of a normal circadian rhythm in airway calibre. The amplitude of the PEFR rhythm is an index of bronchial lability and is thus valuable in monitoringo asthma patients. An amplitude of $>20 \%$ should be a useful screening test for asthma.

Nocturnal or early morning asthma is easily demonstrated physiologically by serial measurements of peak expiratory flow rate (PEFR) at different times of day. ${ }^{1}$ Excessive falls in PEFR in the early morning may have prognostic significance for the clinician ${ }^{23}$ The mechanisms underlying the asthmatic rhythm in airways calibre remain poorly understood ${ }^{4-7}$ Nevertheless, similar rhythms in airway calibre, with much lower amplitudes, have previously been demonstrated in small numbers of normal subjects by repeated measurements of forced expiratory volumes, ${ }^{89}$ or airways resistance. ${ }^{10-12} \mathrm{~A}$ rhythm in PEFR has also been demonstrated in a few normal subjects by Reindl ${ }^{13}$ and Reinberg et al. ${ }^{14}$

These studies have provided relatively little information on the characteristics of the normal rhythm in airway calibre, principally because of the small numbers of subjects recruited. In particular, there is very little information available on the amplitude and phase of the PEFR rhythm in normal subjects. We have, therefore, studied the PEFR rhythm in larger numbers of normal subjects. We

Address for reprint requests: Dr MR Hetzel, Brompton Hospital, Fulham Road, London SW3 6HP. aimed to define the normal rhythm, to compare is with the PEFR rhythms of asthmatic patients, and? to evaluate measurement of the amplitude of the PEFR rhythm as a screening test for asthma.

\section{Methods}

STUDY 1

Normal subjects were recruited who agreed to record their own PEFR at home. They were screenede. by a questionnaire to exclude any cases with an history of cardiac, respiratory, or other currently active disease. They were particularly screened for symptoms of wheezy breathlessness or sputumt production to exclude individuals with asthma and chronic bronchitis. Subjects taking drugs other thano the contraceptive pill were also excluded. They wered subsequently withdrawn from the study if they? regularly failed to achieve a PEFR within twot standard deviations of predicted normal values. ${ }^{15} 10$ The questionnaire also asked for any history of eczema or hayfever, family history of asthma, and 8 smoking history.

PEFR was measured for seven consecutive days on waking, on leaving home for work, on coming 
home in the evening, and at bedtime. At weekends the middle two sets of readings were performed at the same times as had been necessary during the working week. Retired subjects took these readings one hour after waking and at 1800 . On each occasion PEFR was measured three times with a peak flow gauge (Airmed) with a 30 second rest between attempts. All three readings were subsequently analysed individually in the computer programme. Recordings were made in the sitting position and the scale was read to the nearest $51 \mathrm{~min}^{-1}$, the reading accuracy of the instrument. Subjects documented all three readings and the exact time of measurement on a diary chart.

\section{STUDY 2}

Fifteen of the subjects recruited to study 1 were also able to attend for laboratory studies. For five consecutive days (Monday to Friday) they recorded flow-volume loops ${ }^{17}$ at 0930,1200 , and 1730 . Loops were recorded with an Ohio 800 spirometer linked by an Ohio flow-volume converter to a Prime 300 computer which displayed the loops and recorded measurements from it. Three attempts were made on each occasion in the sitting position, with a 30 second rest between attempts.

\section{STUDY 3}

A similar protocol of PEFR measurements to that described in study 1 was carried out for seven days in asthma patients. Criteria for selection of these patients were that they gave a history of variable wheezy breathlessness over short periods of time with improvement after treatment or spontaneously, their sputum production (if any) was insufficient to satisfy the MRC criteria for chronic bronchitis, ${ }^{18}$ and they showed a $>20 \%$ improvement in PEFR or forced expiratory volume in one second $\left(\mathrm{FEV}_{1}\right)$ after inhaled or oral bronchodilator drugs or a $>50 \%$ improvement in PEFR over the course of their hospital admission. They were either studied during convalescence from acute asthma in hospital or at home, immediately after discharge from hospital.

\section{ANALYSIS}

In all three studies data were analysed by the cosinor method of Halberg et al. ${ }^{1920}$ This technique uses a least squares method to test the goodness of fit of the raw data to a sinusoidal waveform. The function:

$$
\left.f(t)=\operatorname{Co}+C \cdot \operatorname{Cos} \frac{(2 \pi t}{T}+\phi\right)+\varepsilon_{t}
$$

where $t=$ time, $f=$ biological variable under study, $\mathrm{Co}=$ constant term or intercept, $\mathrm{C}=$ amplitude, $\phi=$ phase, $T=$ trial period under study, $\varepsilon_{\mathrm{t}}=$ residual error (when mean of $\varepsilon_{t}=0$ and standard deviation of $\varepsilon_{t}=$ standard error of estimate) is fitted to the raw data for different trial periods, $T$. When the subject under study is synchronised to the solar day, $T$ can be assumed to be 24 hours. The rhythm is most conveniently represented as a cosine wave, if the reference point for phase is $0^{\circ}$ or 00.00 hours, since $\cos 0^{\circ}=1$. Halberg's programme was not available to us. A multiple regression programme was, therefore, used to analyse PEFR against time, using the equation:

$$
P E F R=C o+a \cos \frac{(2 \pi t)}{24}+b \sin \frac{(2 \pi t)}{24}
$$

Zero time was taken as 00.00 hours on the first day of the study. Amplitude, $\mathrm{C}$, and phase were determined from the coefficients $\mathrm{a}$ and $\mathrm{b}$ of the cos $(2 \pi \mathrm{t})$ and $\sin (2 \pi \mathrm{t})$ terms in the regression equation.

$\frac{2 \pi}{24} \quad \frac{2 \pi t}{24}$

The amplitude, $\mathrm{C}$, in this mathematical model represents half the difference observed between the highest and lowest values in a complete cycle $\left(360^{\circ}\right.$ or 24 hours). From a clinical viewpoint, however, the peak to trough measurement of the PEFR rhythm-that is, the maximum change in PEFR during the 24 hour cycle-is more important. ${ }^{2}$ For the purposes of the present study we, therefore, preferred to define "amplitude" as the peak to trough measurement and this definition of amplitude is used throughout the results. Thus: peak to trough measurement ("amplitude") $=2 \mathrm{C}=2 \cdot \sqrt{\mathrm{a}^{2}+\mathrm{b}^{2}}$. The phase of the rhythm is identified conventionally as the time of the computed acrophase (peak reading in the 24-hour cycle) and was determined by the equation: phase $\phi^{\circ}=\arctan (-b)$

a

The amplitude (peak to trough measurement) of each individual subject's PEFR rhythm was expressed as a percentage of each individual subject's mean PEFR over the study period to facilitate comparison between normal subjects and asthma patients with widely differing peak flow rates and predicted normal values.

\section{Results}

STUDY 1

Two hundred and twenty-one normal subjects were recruited who satisfied the criteria set by the questionnaire and could achieve a PEFR within the normal range. ${ }^{15} 16$ There were 107 males and 114 females. Mean age was $39 \cdot 1$ years (range $10-84$ years). One 
Table 1 Distribution of rhythmicity and amplitude with age

\begin{tabular}{|c|c|c|c|c|c|c|c|}
\hline Age (yr) & $10-20$ & $21-30$ & $31-40$ & $41-50$ & $51-60$ & $61-70$ & $71+$ \\
\hline Recruited (n) & 46 & 42 & 36 & 39 & 20 & 21 & 17 \\
\hline Significant rhythm (n) & 26 & 31 & 23 & 25 & 16 & 15 & 9 \\
\hline Significant rhythm (\%)* & $56 \cdot 5$ & $73 \cdot 8$ & $63 \cdot 9$ & $64 \cdot 1$ & $80 \cdot 0$ & $71 \cdot 4$ & 52.9 \\
\hline Mean amplitude $\%$ & $8 \cdot 0$ & $7 \cdot 1$ & $6 \cdot 1$ & $8 \cdot 1$ & $8 \cdot 2$ & $13 \cdot 3+$ & $10 \cdot 5$ \\
\hline
\end{tabular}

Grand mean amplitude: $8.3 \%$ of individual mean PEFR ( \pm SD $5.2 \%$ )

*Percentage of subjects in each age group with a significant PEFR rhythm

+Significant difference in mean amplitude from all other age groups $(p<0.05)$

hundred and forty-five subjects had a significant $(\mathrm{p}<0.05)$ rhythm detectable by cosinor analysis. The mean age of this group was 39.7 years (range 10-84 years). Considering each individual subject's amplitude as a percentage of his mean PEFR, the mean amplitude of this rhythmic group was $8 \cdot 3 \%$. The standard deviation of individual results for amplitude measured as a percentage of each individual's mean PEFR was $\pm 5 \cdot 2 \%$. Mean acrophase was at 1526. This rhythmic group comprised 81 males and 64 females. Mean male amplitude was $7.6 \%$ $( \pm$ SD $4.7 \%)$ and mean female amplitude was $8 \cdot 7 \%( \pm$ SD $5.6 \%)$; these differences were not statistically significant.

Table 1 shows the relationship between rhythmicity and age. Subjects have been grouped in decades, except, for convenience, in the under 20 and over 70 year age groups. Reasonable numbers could only be recruited up to the fifth decade because of frequent suspicion of mild cardiac or respiratory disease in older volunteers. The percentage of subjects with a significant rhythm was similar in all age groups (53-80\%). Amplitudes were relatively consistent, irrespective of age, at some $8 \%$ of subjects' individual mean PEFR; although amplitudes in the 61-70 year age group (mean $13.3 \%$ ) were significantly higher $(\mathrm{p}<0.05)$ than in any other age group.

None of the other factors studied in the questionnaire appeared to influence amplitude in these 145 subjects with significant rhythms. Thirty were currently smoking, 37 had previously been regular smokers, and 78 had never smoked. Amplitudes in these three categories were $7 \cdot 8 \%, 8 \cdot 6 \%$, and $8.2 \%$ respectively and were not significantly different from each other. Thirty-nine had a family history of asthma in a blood relative but their mean amplitudes $(7 \cdot 7 \%)$ was not significantly different from the remaining 106 subjects (mean $8.4 \%$ ) without anj family history. Twenty-eight had some history of atopge without asthma but their amplitudes (mean 7.7\% were not significantly different from 117 subjecto with no history of atopy (mean $8 \cdot 3 \%$ ).

STUDY 2

Table 2 contains a comparison of the results of laboratory studies of the flow volume loop, three times a day for five days with PEFR at home for seven days in rhythm detection in the same group of 15 subjects. Eleven showed a significant rhythm in PEFR at home. No single measurement from the flow-volume loop detected significant rhythmicity ing as many of them. Four subjects with no significan rhythm detectable in their home PEFR data did however, demonstrate rhythmicity in at least some components of their flow-volume loops. Individuat subjects varied in those components of the loop in which rhythmicity could be demonstrated, an comparison with the results of PEFR measurementw at home is, therefore, difficult. Table 2 shows the number of subjects with significant rhythmicity ip. each test. For comparison the mean amplitude and acrophase quoted for each test are those of the whole group, even though some subjects did not achieve significant rhythmicity in a given test. The mean acrophase for PEFR at home in these 15 normal subjects (1533) can be compared with acrophases ranging from 0956-1531 in differen components of the flow-volume loop.

Table 2 Rhythmicity at home and in the laboratory $(n=15)$

\begin{tabular}{|c|c|c|c|c|c|c|c|c|c|c|c|}
\hline & \multirow{2}{*}{$\begin{array}{l}\text { Home } \\
(0700-2300)\end{array}$} & \multicolumn{10}{|c|}{ L aboratory (0930-1730) } \\
\hline & & $P E F R$ & $F E V_{1}$ & $\dot{V}_{m 50}$ & $\dot{V}_{m 25}$ & $F V C$ & $P I F$ & $F I V_{1}$ & $\dot{V}_{I m 50}$ & $\dot{V}_{I m 25}$ & FIC \\
\hline $\begin{array}{l}\text { Significant rhythmn (n) } \\
\text { Mean amplitude } \%\end{array}$ & $\begin{array}{l}11 \\
5 \cdot 2\end{array}$ & $\begin{array}{c}7 \\
9 \cdot 5\end{array}$ & $\begin{array}{c}8 \\
6 \cdot 9\end{array}$ & $\begin{array}{c}6 \\
13 \cdot 5\end{array}$ & $\begin{array}{c}4 \\
16 \cdot 9\end{array}$ & $\begin{array}{c}7 \\
4 \cdot 3\end{array}$ & $\begin{array}{c}2 \\
15 \cdot 0\end{array}$ & $\begin{array}{c}0 \\
18 \cdot 6\end{array}$ & $\begin{array}{c}3 \\
20 \cdot 5\end{array}$ & $\begin{array}{c}1 \\
17 \cdot 2\end{array}$ & $\begin{array}{c}7 \\
4 \cdot 2\end{array}$ \\
\hline Acrophase (hours) & $15 \cdot 33$ & $10 \cdot 09$ & $15 \cdot 31$ & $13 \cdot 53$ & $14 \cdot 05$ & $13 \cdot 32$ & $10 \cdot 43$ & $13 \cdot 32$ & $09 \cdot 56$ & $10 \cdot 46$ & $13 \cdot 44$ \\
\hline
\end{tabular}

PEFR = peak expiratory flow rate; $\mathrm{FEV}_{1}=$ forced expiratory volume in one second; $\dot{\mathrm{V}}_{\mathrm{m} 50}=$ maximal expiratory flow rate at $50 \%$ vital capacity $\dot{\mathrm{V}}_{\mathrm{m} 25}=$ maximal expiratory flow rate at $25 \%$ vital capacity; FVC $=$ forced vital capacity; PIF $=$ peak inspiratory flow rate; FIV ${ }_{1}$ forced inspiratory volume in one second; $\dot{V}_{\text {Im } 50}=$ maximal inspiratory flow rate at $50 \%$ vital capacity; $\dot{V}_{i m 25}=$ maximal inspiratory flow rate a $25 \%$ vital capacity; FIC = forced inspiratory capacity. 


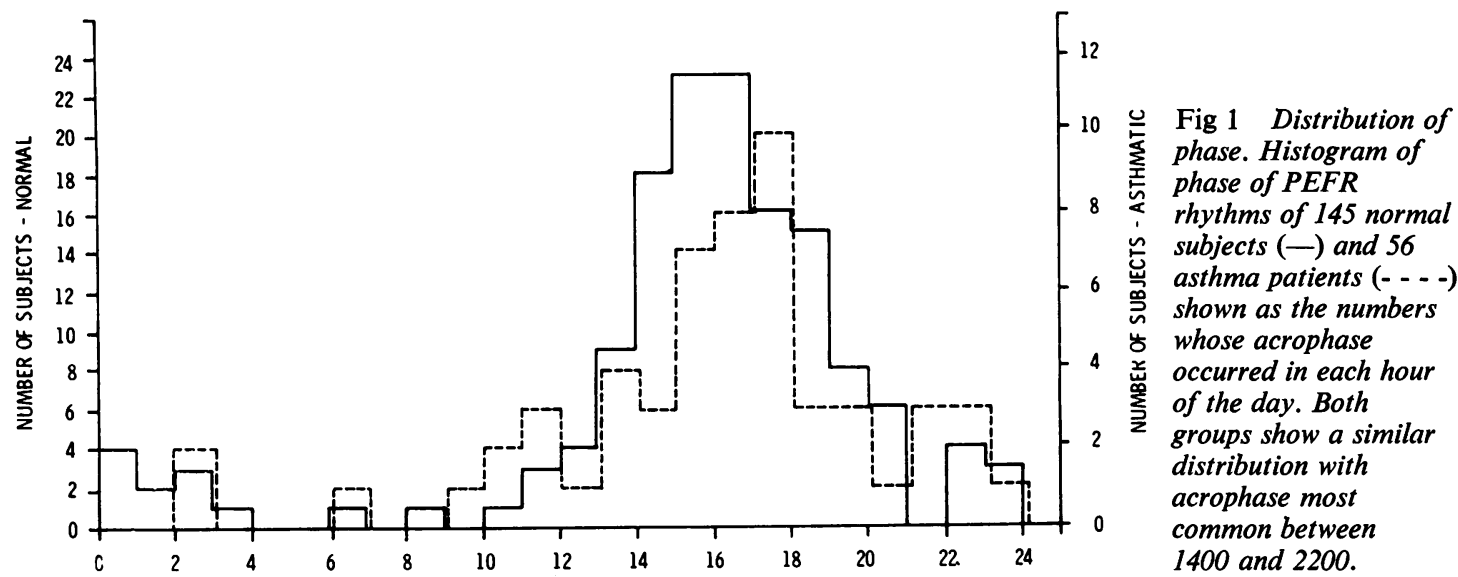

TIME - HOURS

STUDY 3

The mean age of the 56 asthma patients was $41 \cdot 4$ years (range 16-67 years). There were 20 males and 36 females. Twenty-eight had positive skin tests to common allergens. Their duration of asthma symptoms ranged from six months to 42 years (mean 18 years). Fifty patients were treated with corticosteroids, the remainder took bronchodilator drugs only. All showed significant rhythms in PEFR. Mean amplitude was $50.9 \%$ of patients' individual mean PEFR ( \pm SD $41 \cdot 7 \%$ ). Mean acrophase was at 1557 hours.

Figure 1 compares the phases of the PEFR rhythms of the 56 asthma patients with the 145 normal subjects in whom a significant rhythm was demonstrated in study 1; results are shown as a histogram of the number of subjects whose acrophase occurred in each hour of the day. The distribution of phase was similar between normal subjects and asthma patients so that the majority had an acrophase between 1400 and 2200 hours.
Figure 2 uses the same format to illustrate the distribution of the estimated acrophases in the remaining 76 normal subjects in whom rhythmicity could not be demonstrated at a statistically significant level in study 1 . The distribution of these estimates for phase nevertheless appeared similar to that shown in fig 1 with the majority of these 76 subjects having an acrophase between 1300 and 1700 .

Figure 3 illustrates the normal and asthmatic PEFR rhythms as a cosinor summary. ${ }^{20}$ The mean rhythms of the 145 normal subjects with significant rhythmicity and the 56 asthma patients are shown as vectors with their length proportional to their amplitude and their angle indicating their phase (time of the acrophase or highest point in the cycle). The error ellipses around the ends of these vectors show the $95 \%$ confidence limits for amplitude and phase. The dotted lines indicate overlap of the ellipses for phase, which is not therefore significantly different between normal subjects and asthma patients. The arrows indicate the $95 \%$ confidence limits for the

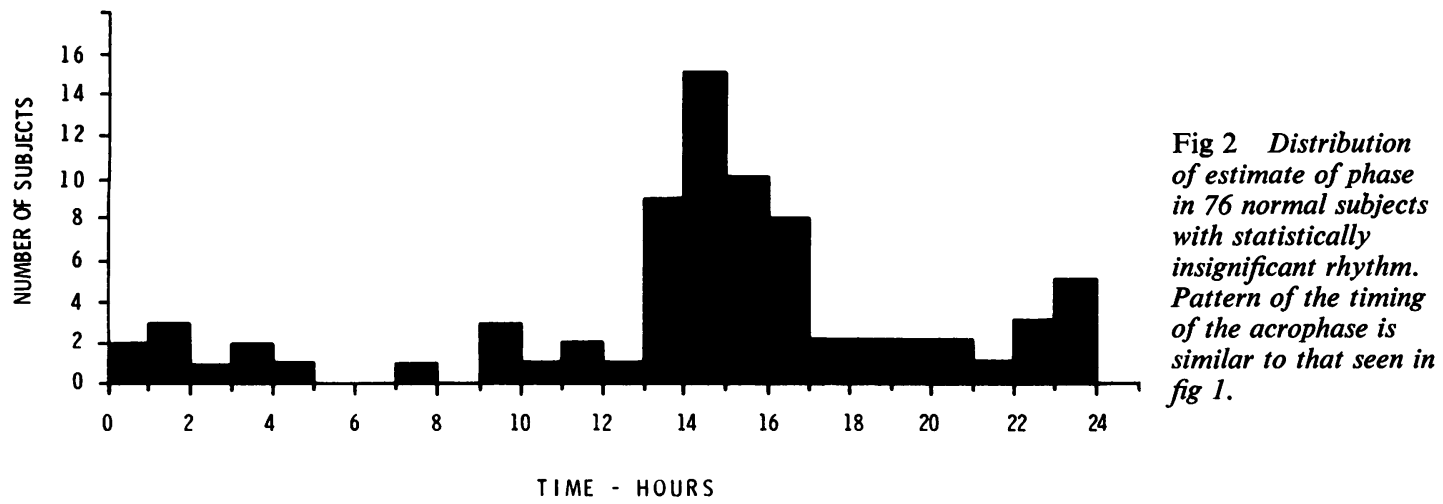




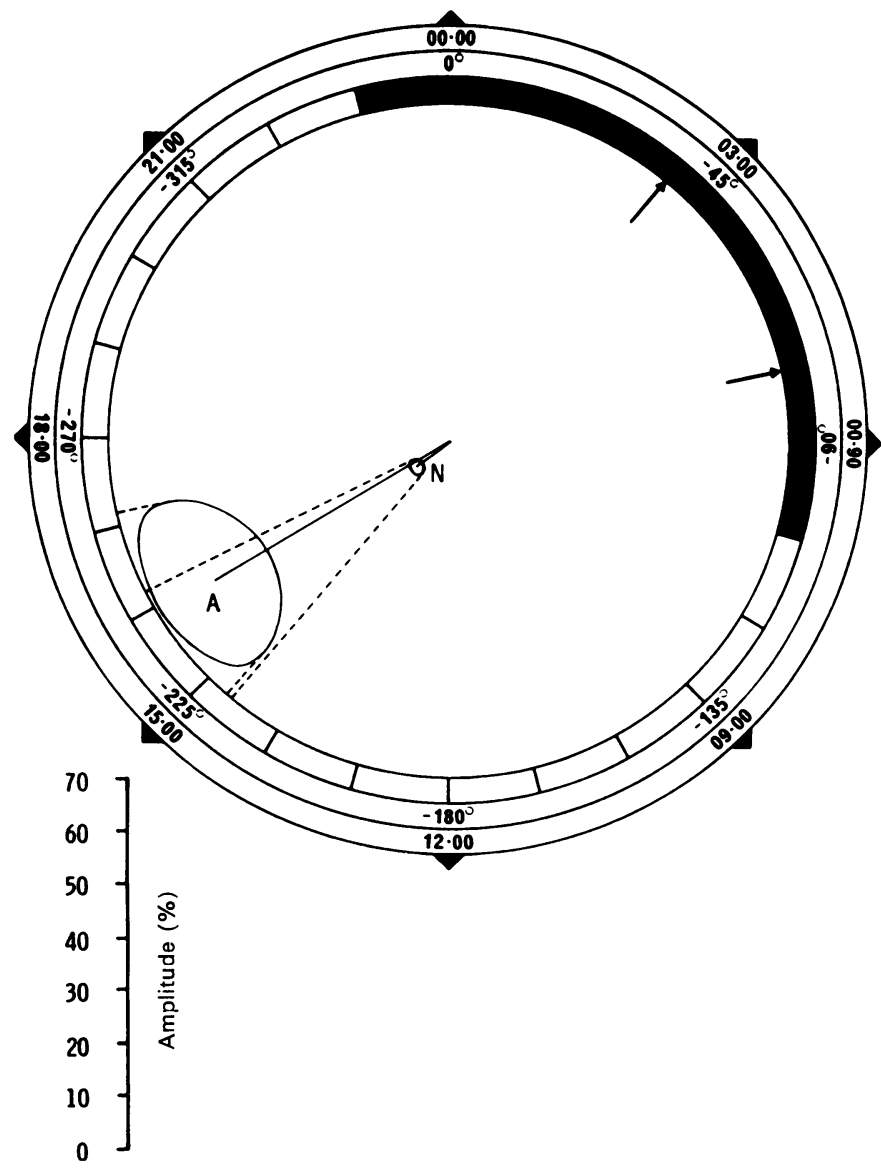

Fig 3 Cosinor summary of normal and asthmatic rhythms. Rhythms are shown as vectors with length proportional to amplitude and angle indicating phase. Error ellipses enclose the $95 \%$ confidence limits for amplitude and phase. Dotted lines show 0 overlap for phase-thus mean acrophases of 1526 in normal subjects and 1557 in asthma patients are not significantly different. Mean amplitude in asthma patients $(50.9 \%)$ is, however, significantly higher than in normal subjects (mean $8.3 \%$ ). $A=$ asthma patients $(n=56)$, $N=$ normal subjects $(n=145), \uparrow=$ range of bathyphase, $\mathbf{\square}=$ lights off.

bathyphase (lowest point in the cycle) for the normal and asthmatic rhythms, which ranged between 0240 and 0515.

\section{Discussion}

Our results show that a low amplitude circadian rhythm in airway calibre can be demonstrated in the majority of normal subjects (145 of 221 or $65.6 \%$ ) by measuring PEFR with a very simple instrument. Moreover, in the remaining 76 normal subjects, in whom rhythm detection did not achieve statistical significance, the computed estimates of the phase of their rhythms nevertheless showed a very similar distribution (fig 2) to that seen in the subjects with significant rhythmicity (fig 1). This similarity would not have been expected if results in these 76 subjects were an artefact, from biological noise alone, without any underlying periodicity. We, therefore, suggest that all normal subjects have a circadian rhythm in PEFR but, in the minority of those studied, amplitude was too low for rhythn? detection with the peak flow gauge.

Study 2 compared the performance of the peak flow gauge at home with laboratory measurement of the flow-volume loop in rhythm detection Because subjects could only be studied over abou? half their waking day in the laboratory, use of more sophisticated apparatus in study 2 proved no more effective than the peak flow gauge at home $N$ Moreover, computation of amplitude and phase्w may have been less accurate as it was based ono readings which were less well distributed over the 24-hour cycle. Study 2 did, however, demonstrate्ष rhythmicity in four subjects in whom significan? PEFR rhythms were not demonstrable at home.0 This is further evidence in favour of the view thas all normal subjects have a rhythm in airwas calibre.

The cosinor analysis ${ }^{1920}$ is commonly used by chronobiologists in the detection of circadian rhythms in biological data. It has the advantage thap 
a relatively small number of measurements are required during each cycle of the rhythm, provided these are well distributed throughout the cycle. The exact time of readings is also taken into account, so that any readings taken later than intended can still be used without impairing the accuracy of rhythm detection. This method also determines the phase of the rhythm. The majority of previous studies of rhythms in airway calibre in normal subjects $^{8-12}$ have simply compared readings at different times of day, in some cases for a single day only, and have usually used an analysis of variance to assess the significance of the difference in results with the time of day. This type of approach can only give a rough idea of the phase of the rhythm and frequent readings are required to judge it with any accuracy. Nevertheless, to validate further the cosinor method we reanalysed the data of the first 80 subjects recruited to study 1 as four blocks of readings at the four times of measurement each day and used an analysis of variance to assess the difference between these blocks of readings. A statistically significant difference with time of day was only found in those subjects whose data had revealed significant rhythmicity with cosinor analysis and vice versa.

Results indicated a mean amplitude in the 145 normal subjects with significant rhythmicity of $8.3 \%$ of individual mean PEFR with a sample standard deviation of $5.2 \%$. Thus, as our series was reasonably large, normal subjects would seem unlikely to exceed an amplitude of $20 \%$ of mean PEFR. Amplitude of this magnitude should be easily recognisable from simple recordings of PEFR against time without computer analysis. An amplitude in excess of $20 \%$ of mean PEFR would, therefore, be a convenient and realistic threshold above which subjects are unlikely to be asthmatic. Now that the mini peak flow meter ${ }^{21}$ is available and has been shown to be reliable, ${ }^{22}$ measurement of the amplitude of the PEFR rhythm should be valuable in clinical practice in the diagnosis of asthma in cases who are in remission. Patients could record their own data at home as they can be shown to keep accurate records with minimal training. ${ }^{23}$ The results for phase in our studies suggest that readings on waking, at 16.00 hours, and at bedtime would be a convenient protocol of measurements which would give a good approximation of the amplitude of the PEFR rhythm.

No factors were found which influenced amplitude in the PEFR rhythm of normal subjects in study 1. There was some indication of a possible trend towards increasing amplitude with age but this was only significant in the 61-70 year age group. We suspect this was an artefact, however, since numbers were smaller in the older age groups and we were less confident of their normality since predicted normal PEFR could only be estimated by extrapolation of Cotes' data ${ }^{15}$ in the over 60 s. Thus we found a fairly narrow range for amplitude in normal subjects. The 56 asthma patients recruited to study 3 were comparable to the 145 rhythmic normal subjects for age and sex and the phases of their rhythms were strikingly similar. The asthmatic rhythm was distinguished only by its greater amplitude. The phenomenon of nocturnal and early morning asthma, therefore, probably results from amplification of a normal circadian rhythm in airway calibre by the abnormally labile asthmatic airways.

We conclude that measurement of the amplitude of the PEFR rhythm is a valuable clinical tool in the diagnosis and management of asthma. Since it appears to relate to bronchial lability it has particular value in detection of unstable asthma as previously reported in its association with sudden asthma death. ${ }^{23}$ In addition to increasing the mean PEFR, reduction of the amplitude of the PEFR rhythm should also be an objective of treatment. Measurement of PEFR amplitude, therefore, merits consideration in trials of new drugs for asthma.

This study was supported by a grant from the Board of Governors of Brompton Hospital.

\section{References}

1 Turner-Warwick M. On observing patterns of airflow obstruction in chronic asthma. Br J Dis Chest 1977; 71:73-86.

2 Hetzel MR, Clark TJH, Branthwaite MA. Asthma: analysis of sudden deaths and ventilatory arrests in hospital. Br Med J 1977; 1:808-11.

3 Bateman JRM, Clarke SW. Sudden death in asthma. Thorax 1979; 34:40-4.

4 Mills JN. Human circadian rhythms. Physiol Rev $1966 ; 46: 128-71$.

5 Soutar CA, Costello J, Ijaduola O, Turner-Warwick $M$. Nocturnal and early morning asthma. Relationship to plasma corticosteroids and response to cortisol infusion. Thorax 1975; 30:436-40.

6 Soutar CA, Carruthers M, Pickering CA. Nocturnal asthma and urinary adrenaline and noradrenaline excretion. Thorax 1977; 32:677-83.

7 Hetzel MR, Clark TJH. Does sleep cause nocturnal asthma? Thorax 1979; 34:749-54.

8 Lewinsohn HC, Capel LH, Smart J. Changes in forced expiratory volumes throughout the day. Br Med J 1960; 1:462-4.

9 Guberan E, Williams MK, Walford J, Smith MM. Circadian variation of FEV in shift workers. $B r J$ Ind Med 1969; 26:121-5.

10 McKerrow CB, McDermott M, Gilson JC, Schilling RSP. Respiratory function during the day in cotton 
workers: a study of byssinosis. Br J Ind Med 1958; 15:75-83.

11 McDermott M. Diurnal and weekly cyclical changes in lung airways resistance. $J$ Physiol 1966; 186: 90P-2P.

12 Kerr HD. Diurnal variation of respiratory function independent of air quality. Arch Environ Health 1973; 26:144-52.

13 Reindl K. Doktorarbeit 1967. Cited: Reindl K, Falliers C, Halberg F, Chai H, Hillman D, Nelson W. Circadian acrophase in peak expiratory flow rate and urinary electrolyte excretion of asthmatic children. Rass Neur Veget 1970; 23:5-26.

14 Reinberg A, Gervais P. Circadian rhythms in respiratory functions with special reference to human chronophysiology and chronopharmacology. Bull Physiopathol-Respir 1972; 8:663-77.

15 Cotes JE. Lung function. Assessment and application in medicine. Third edition. Oxford: Blackwell, 1975:383-9.

16 Godfrey S, Kamburoff PL, Nairn JR. Spirometry, lung volumes and airways resistance in normal children aged 5-18 years. Br J Dis Chest 1970; 64:15-24.
17 Bass $\mathrm{H}$. The flow volume loop: normal standards and abnormalities in chronic obstructive pulmonary disease. Chest 1973; 63:171-6.

18 Medical Research Council Committee on the aetiology of chronic bronchitis. Definition anc classification of chronic bronchitis for clinical andepidemiological purposes. Lancet 1965 ; 1 :775-9.

19 Halberg F, Diffley M, Stein M, Panofsky H, Adkins $\vec{\overrightarrow{ }}$ G. Computer techniques in the study of biologicab rhythms. Ann NY Acad Sci 1964; 115:695-720.

20 Halberg F, Engeli M, Hamburger C, Hillman $D_{\omega}^{\times}$ Spectral resolution of low frequency, small amplitude rhythms in excreted 17 ketosteroids; probable $\overrightarrow{0}$ androgen induced circaseptan desynchronization Acta Endocrinol 1965; Suppl no 103:1-54.

21 Wright RM. A miniature Wright peak-flow meter? Br Med J 1978; 2:1627-8.

22 Perks WH, Tams IP, Thompson DA, Prowse K.O An evaluation of the mini-Wright peak flow meter Thorax 1979; 34:79-81.

23 Hetzel MR, Williams IP, Shakespeare RM. Can patients record peak flow rate reliably? Lanceko 1979; 1:597-9. 